Original Research Paper

\title{
Odds Exponential Log Logistic Distribution: Properties and Estimation
}

\author{
${ }^{1}$ Rosaiah Kanaparthi, ${ }^{2}$ Gadde Srinivasa Rao, \\ ${ }^{3}$ Kruthiventi Kalyani and ${ }^{3}$ Devireddy Charana Udaya Sivakumar \\ ${ }^{I}$ Department of Statistics, Acharya Nagarjuna University, Guntur - 522 510, India \\ ${ }^{2}$ Department of Statistics, The University of Dodoma, P.O. Box: 259, Tanzania \\ ${ }^{3}$ UGC BSR Fellows, Department of Statistics, Acharya Nagarjuna University, Guntur - 522 510, India
}

Article history

Received: 06-09-2016

Revised: 02-01-2017

Accepted: 17-01-2017

Corresponding author:

Gadde Srinivasa Rao

Department of Statistics, The

University of Dodoma, P.O.

Box: 259, Tanzania

Email: gaddesrao@yahoo.com

\begin{abstract}
We propose a distribution called Odds Exponential Log Logistic Distribution (OELLD), which is an odds family of distribution. Its hazard rate is an increasing and decreasing function based on the value of the parameter. Explicit expressions for the ordinary moments, L-moments, quantile, generating functions, Bonferroni Curve, Lorenz Curve, Gini's index and order statistics are derived. The parameters of the proposed distribution are estimated by using maximum likelihood method and also illustrated by a lifetime data set.
\end{abstract}

Keywords: Exponential Family, Log Logistic Distribution, ML Estimation, 2010 Mathematics Subject Classification: 62E10, 62F10

\section{Introduction}

In describing the real world phenomena, the distributions are very much useful. In spite of the fact that a number of distributions are developed, always there is a scope for introducing distributions, analysing their properties to use them for fitting real world scenarios. Hence, we are always developing new and not rigid distributions. In recent works, new distributions are defined by introducing one or more parameters to the distribution functions. The addition of parameters to the distribution functions makes the distribution richer and more flexible for modelling life time data. But, at the same time adding of too many parameters to the distribution make inferential aspect more complicated. Proportional Odds Model (POM), Proportional Hazard Model (PHM), Proportional reversed hazard model (PRHM), Power Transformed Model (PTM) are some of the models originated from this idea to add a shape parameter. For such models, a few pioneering works are by Box and Cox (1964; Cox, 1972; Marshall and Olkin, 1997; Gupta and Kundu, 1999; Kantam and Rao, 2002; Ashkar and Mahdi, 2006; Rosaiah et al., 2006; Gupta and Gupta, 2007; Aljarrah et al., 2014; Tahir et al., 2015; El-Damcese et al., 2015) and the references therein. Many distributions have been developed in recent years that involve the logit of the beta distribution. Using the generalized class of beta distribution, the distribution function $(d f)$ for this class of distributions for the random variable $X$ is generated by applying the inverse of the $d f$ of $X$ to a beta distributed random variable:

$$
F(x)=\frac{1}{B(\alpha, \beta)} \int_{0}^{G(x)} t^{\alpha-1}(1-t)^{\beta-1} d t ;>0, \alpha, \beta>0
$$

where, $G(x)$ is the $d f$ of other distribution. This class of distributions has not only generalized the beta distribution but also supplemented the parameter(s) to it. Among this class of distributions are, the beta-Normal Eugene et al. (2002); beta-Gumbel Nadarajah and Kotz (2004); beta-Weibull Famoye et al. (2005); betaExponential Nadarajah and Kotz (2006); beta-Laplace Kozubowski and Nadarajah (2008) and beta-Pareto Akinsete et al. (2008), beta-Rayleigh Akinsete and Lowe (2009). Number of useful statistical properties arising from these distributions and their applications to real life data has been discussed in the literature.

Alzaatreh et al. (2013) has proposed a new generalized exponential family of distributions, known as $\mathrm{T}-\mathrm{X}$ family and the cumulative distribution function (cdf) is defined as:

$F(x ; \lambda, \theta)=\int_{a}^{W\left(F_{\theta}(x)\right)} f_{\lambda}(t) d t$

where, the random variable $T, X \in[a, b]$, for $-\infty<a, b<$ $\infty$ and $W\left(F_{\theta}(x)\right)$ be a function of the cdf $F_{\theta}(x)$, so that $W\left(F_{\theta}(x)\right)$ should satisfies the following conditions: 
- $W\left(F_{d}(x)\right) \in[a, b]$

- $W\left(F_{d}(x)\right)$ is differentiable and monotonically nondecreasing

- $\quad W\left(F_{d}(x)\right) \rightarrow a$ as $x \rightarrow \infty$ and $W\left(F_{d}(x)\right) \rightarrow b$ as $x \rightarrow \infty$

We defined a generalized class of any distribution having positive support. Taking $W\left(F_{\theta}(x)\right)=\frac{F_{\theta}(x)}{1-F_{\theta}(x)}$, the odds function, the cdf of the proposed distribution is:

$F(x ; \lambda, \theta)=\int_{0}^{\frac{F_{\theta}(x)}{1-F_{\theta}(x)}} f_{\lambda}(t) d t$

The support of the resulting distribution will be that of $F_{\theta}(\cdot)$.

Here, $\quad \frac{F_{\theta}(x)}{1-F_{\theta}(x)}=\frac{F_{\theta}(x)}{\bar{F}_{\theta}(x)} \rightarrow \infty, \quad$ as $\quad x \rightarrow \infty$. The corresponding distribution is the generalization to the base distribution. This type of distributions is named as Odds Family of Distributions (OFD).

In this study, we take upper and lower incomplete gamma functions are $\Gamma(p, x)=\int_{x}^{\infty} w^{p-1} e^{-w} d w \quad$ and $\gamma(p, x)=\int_{0}^{x} w^{p-1} e^{-w} d w$, for $x \geq 0, p>0 \quad$ respectively. Also, the $j$ th derivatives with respect to $p$ are denoted by $\Gamma^{(j)}(p, x)=\int_{x}^{\infty}(\ln w)^{j} w^{p-1} e^{-w} d w \quad$ and $\quad \gamma^{(j)}(p, x)=$ $\int_{0}^{x}(\ln w)^{j} w^{p-1} e^{-w} d w$, for $x \geq 0, p>0$ respectively.

In this study, we choose particular function $f_{\lambda}(t)=$ $(1 / \lambda) \mathrm{e}^{-t / \lambda}$, i.e., the exponential distribution and the $\log$ logistic distribution $F_{\sigma ; \theta}(x)=\frac{x^{\theta}}{\sigma^{\theta}+x^{\theta}}$, given in Equation

2. Hence, we name this distribution as Odds Exponential Log Logistic Distribution (OELLD). The development of new distribution is presented in Section 2. A study of statistical properties along with moments, L-moments, quantile, generating functions, Bonferroni Curve, Lorenz Curve, Gini's index and order statistics and reliability of the new distribution is provided in Section 3. In Section 4, ML estimation of parameters is discussed and a real life data set has been analysed and compared the results with other fitted distributions. In Section 5, we present the concluding remarks.

\section{The Odds Exponential Log logistic Distribution (OELLD)}

The cumulative distribution function (cdf) of the new Odds Exponential Log Logistic Distribution (OELLD) is defined as:

$$
F(x)=\int_{0}^{\frac{F_{\sigma, \theta}(x)}{1-F_{\sigma, \theta}(x)}} f_{\lambda}(t) d t
$$

where:

$$
F_{\sigma ; \theta}(x)=\frac{x^{\theta}}{\sigma^{\theta}+x^{\theta}} \text { and } f_{\lambda}(x)=\frac{1}{\lambda} e^{-(x / \lambda)}, x>0, \quad \sigma, \theta, \lambda>0
$$

so that:

$F(x ; \lambda, \sigma, \theta)=\int_{0}^{\left(\frac{x}{\sigma}\right)^{\theta}} \frac{1}{\lambda} e^{-(x / \lambda)} d x=1-e^{-\frac{1}{\lambda}\left(\frac{x}{\sigma}\right)^{\theta}}$

Also the probability density function (pdf) of the OELLD is:

$f(x ; \lambda, \sigma, \theta)=\frac{\theta}{\lambda \sigma^{\theta}} x^{\theta-1} e^{-\frac{1}{\lambda}\left(\frac{x}{\sigma}\right)^{\theta}}, x>0, \quad \sigma, \theta, \lambda>0$

with range $(0, \infty)$, the following Fig. 1 shows the pdfs, Fig. 2 shows the distribution function and Fig. 3 hazard rate and reversed hazard rate for different values of $\lambda, \sigma$ and $\theta$.

\section{Statistical Properties}

\section{Distribution Function Limits}

Since the cdf of OELLD is $F(x ; \lambda, \sigma, \theta)=1-e^{-\frac{1}{\lambda}\left(\frac{x}{\sigma}\right)^{\theta}}$, we have:

$$
\lim _{x \rightarrow 0} F(x ; \lambda, \sigma, \theta)=0 \text { and } \lim _{x \rightarrow \infty} F(x ; \lambda, \sigma, \theta)=1
$$

\section{Some Statistical Measures of OELLD}

The mean and medians are:

$$
\mu_{1}^{\prime}=E(X)=\int_{0}^{\infty} x f(x ; \lambda, \sigma, \theta) d x=\sigma \lambda^{1 / \theta} \Gamma\left(\frac{1}{\theta}+1, \lambda\right)
$$

and:

$$
0.5=\int_{0}^{M} f(x ; \lambda, \sigma, \theta) d x=1-e^{-\frac{1}{\lambda}\left(\frac{M}{\sigma}\right)^{\theta}} \Rightarrow M=\sigma[\lambda \ln 2]^{1 / \theta}
$$

The mode is:

$$
\begin{aligned}
& \frac{d}{d x} \ln f(x ; \lambda, \sigma, \theta) \\
& =\frac{d}{d x}\left\{-\ln \lambda+\ln \theta-\theta \ln \sigma+(\theta-1) \ln \left(\frac{x}{\sigma}\right)-\frac{1}{\lambda}\left(\frac{x}{\sigma}\right)^{\theta}\right\}=0 \\
& \Rightarrow \text { Mode }=\left[\frac{(\theta-1) \lambda \sigma^{\theta}}{\theta}\right]^{1 / \theta}
\end{aligned}
$$



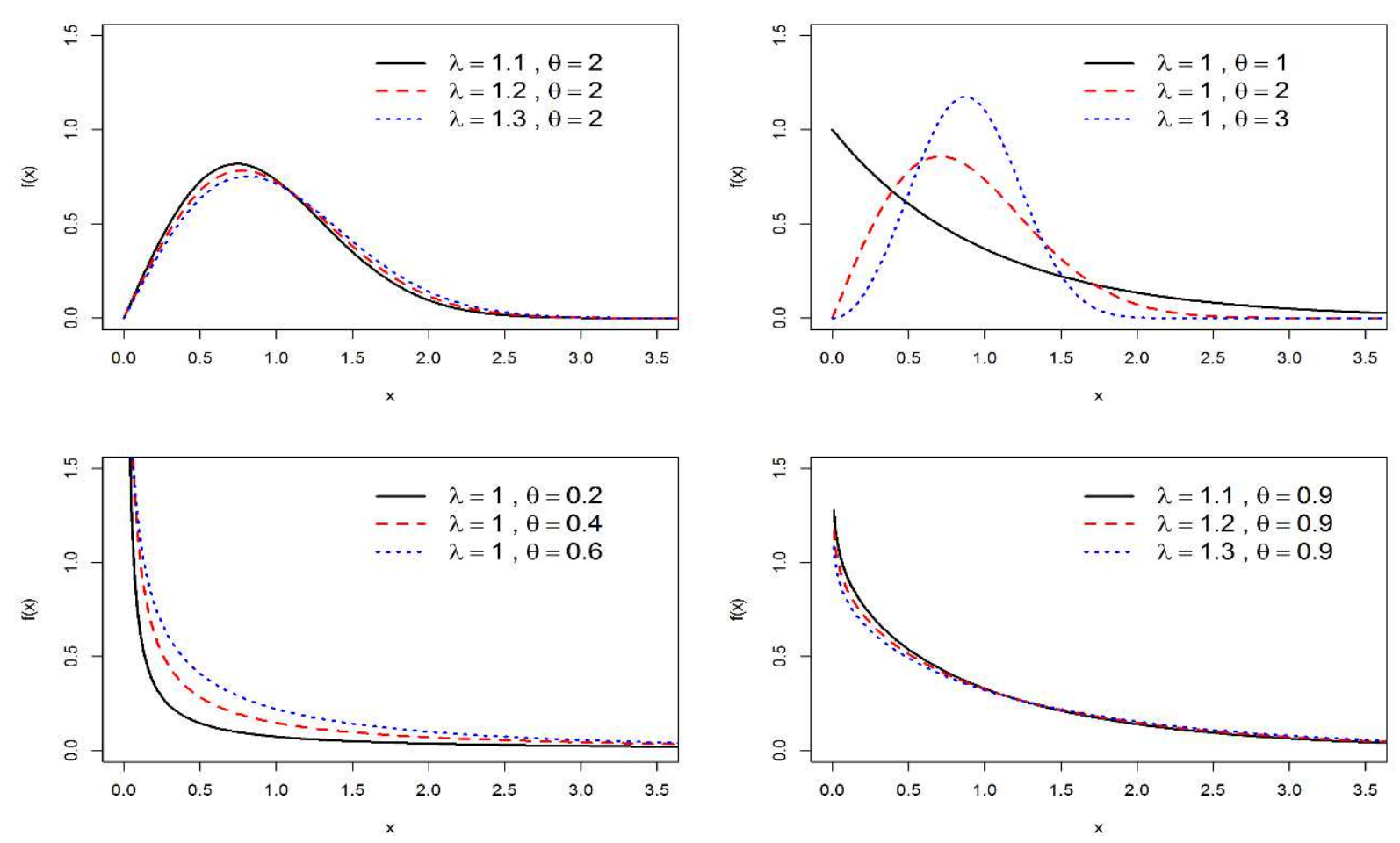

Fig. 1. The probability density function of OELLD for different $\lambda$ and $\theta$ at $\sigma=1$
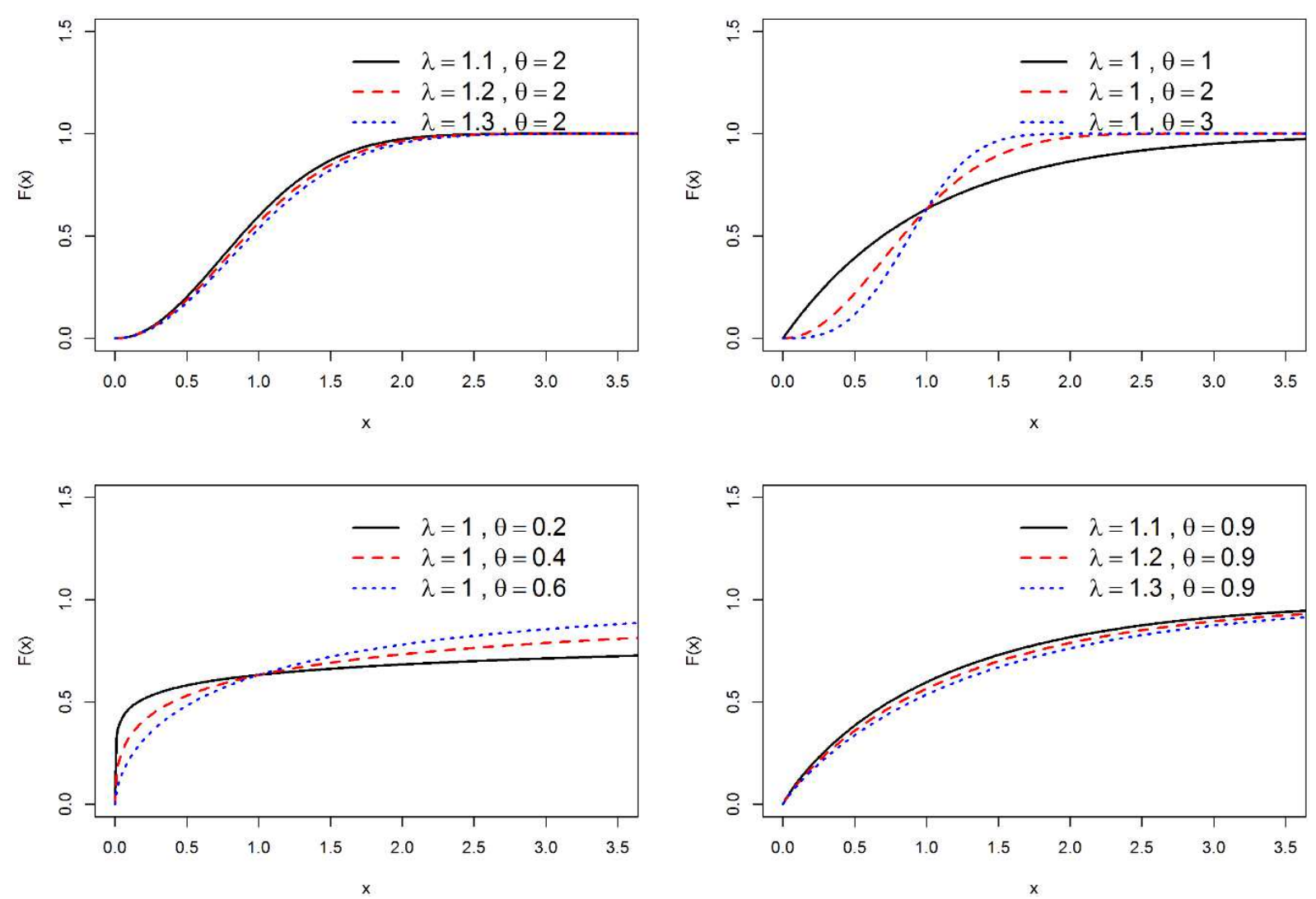

Fig. 2. The distribution function of OELLD for different $\lambda$ and $\theta$ at $\sigma=1$ 
The $r^{\text {th }}$ order raw moment is:

$$
\begin{aligned}
& \mu_{r}^{\prime}=E\left(X^{r}\right)=\int_{0}^{\infty} x^{r} \frac{\theta}{\lambda \sigma}\left(\frac{x}{\sigma}\right)^{\theta-1} e^{-\frac{1}{\lambda}\left(\frac{x}{\sigma}\right)^{\theta}} d x \\
& =\lambda^{r / \theta} \sigma^{r} \Gamma\left(\frac{r}{\theta}+1, \lambda\right)
\end{aligned}
$$

The Skewness $\left(k_{1}\right)$ is:

$$
k_{1}=\frac{\mu_{3}^{2}}{\mu_{2}^{3}} \Rightarrow k_{1}=\frac{\left\{\Gamma\left[\frac{3}{\theta}+1, \lambda\right]\right\}^{2}}{\left\{\Gamma\left[\frac{2}{\theta}+1, \lambda\right]\right\}^{3}}
$$

The Kurtosis $\left(k_{2}\right)$ of the OELLD is:

$$
k_{2}=\frac{\mu_{4}}{\mu_{2}^{2}} \Rightarrow k_{2}=\frac{\Gamma\left[\frac{4}{\theta}+1, \lambda\right]}{\left\{\Gamma\left[\frac{2}{\theta}+1, \lambda\right]\right\}^{3}}
$$

Moment generating function (MGF):

$$
\begin{aligned}
& M_{x}(t)=E\left[e^{t x}\right]=\mathrm{E}\left[1+t x+\frac{t^{2} x^{2}}{2 !}+\frac{t^{3} x^{3}}{3 !}+\ldots+\frac{t^{r} x^{r}}{r !}+\ldots\right] \\
& =\sum_{r=0}^{\infty} \frac{t^{r}}{r !} \mu_{r}^{\prime}=\sum_{r=0}^{\infty} \frac{t^{r}}{r !} \lambda^{r / \theta} \sigma^{r} \Gamma\left(\frac{r}{\theta}+1, \lambda\right)
\end{aligned}
$$

$$
\text { Characteristic Function (C.F.): }
$$

$$
\phi_{x}(t)=E\left[e^{i t x}\right]=\sum_{r=0}^{\infty} \frac{(i t)^{r}}{r !} \lambda^{r / \theta} \sigma^{r} \Gamma\left(\frac{r}{\theta}+1, \lambda\right)
$$

$$
\text { Cumulant Generating Function (C.G.F): }
$$

$$
K_{x}(t)=\ln _{e}\left(M_{x}(t)\right)=\ln _{e}\left[\sum_{r=0}^{\infty} \frac{t^{r}}{r !} \sigma^{r / \theta} \sigma^{r} \Gamma\left(\frac{r}{\theta}+1, \lambda\right)\right]
$$

\section{Mean Deviation}

The mean deviation about the mean and the mean deviation about the median are:

$$
\begin{aligned}
& M d(\text { about mean })=\int_{0}^{\infty}|x-\mu| f(x) d x \\
& =2 \mu F(\mu)-2 \mu+2 \int_{\mu}^{\infty} x f(x ; \lambda, \sigma, \theta) d x
\end{aligned}
$$

We have:

$$
\begin{aligned}
& \int_{\mu}^{\infty} x f(x ; \lambda, \sigma, \theta) d x \\
& =\int_{\mu}^{\infty} x \frac{\theta}{\lambda \sigma}\left(\frac{x}{\sigma}\right)^{\theta-1} e^{-\frac{1}{\lambda}\left(\frac{x}{\sigma}\right)^{\theta}} d x=\lambda^{1 / \theta} \sigma \Gamma\left(\frac{1}{\beta}+1, \frac{\mu^{\theta}}{\lambda \sigma^{\theta}}\right)
\end{aligned}
$$

Therefore, mean deviation about mean is:

$$
\begin{gathered}
M d_{\mu}=2 \mu\left[1-e^{-\frac{1}{\lambda}\left(\frac{\mu}{\sigma}\right)^{\theta}}\right]-2 \mu+2 \sigma \lambda^{1 / \theta} \Gamma\left(\frac{1}{\theta}+1, \frac{\lambda \mu^{\theta}}{\sigma^{\theta}}\right) \\
M d(\text { about median })=\int_{0}^{\infty}|x-M| f(x ; \lambda, \sigma, \theta) d x
\end{gathered}
$$

$$
\begin{aligned}
& M d_{M}=\int_{0}^{M}(M-x) f(x ; \lambda, \sigma, \theta) d x \\
& +\int_{M}^{\infty}(x-M) f(x ; \lambda, \sigma, \theta) d x \\
& =M F(M)-\int_{0}^{M} x f(x ; \lambda, \sigma, \theta) d x \\
& +\int_{M}^{\infty} x f(x ; \lambda, \sigma, \theta) d x-M\{1-F(M)\}
\end{aligned}
$$

Therefore, mean deviation about median is:

$$
M d_{M}=-\mu+2 \sigma \lambda^{1 / \theta} \Gamma\left(\frac{1}{\theta}+1, \frac{\lambda M^{\theta}}{\sigma^{\theta}}\right)
$$

\section{Conditional Moments}

In reliability theory, the residual life and the reversed residual life play an important role.

The $r^{\text {th }}$ order raw moment for the residual life is:

$$
\mu_{r}^{\prime}(t)=E\left[(X-t)^{r} / X>t\right]=\frac{1}{\bar{F}(t)} \int_{t}^{\infty}(x-t)^{r} f(x) d x
$$

Let $x^{\theta}=u$, then:

$$
\begin{aligned}
& \mu_{r}^{\prime}(t)=\frac{1}{\lambda \sigma^{\theta} e^{-\frac{1}{\lambda}\left(\frac{t}{\sigma}\right)^{\theta}} \int_{t^{\theta}}^{\infty}\left(u^{1 / \theta}-t\right)^{r} e^{-u / \lambda \sigma^{\theta}} d u} \\
& =\frac{1}{\lambda \sigma^{\theta} e^{-\frac{1}{\lambda}\left(\frac{t}{\sigma}\right)^{\theta}} \sum_{j=0}^{r}(-1)^{j}\left(\begin{array}{l}
r \\
j
\end{array}\right) t^{r-j} \sigma^{j} \lambda^{j / \theta} \Gamma\left(\frac{j}{\theta}+1, \frac{\lambda t^{\theta}}{\sigma^{\theta}}\right)}
\end{aligned}
$$

The $r^{\text {th }}$ order raw moment for the reversed residual life is: 


$$
\begin{aligned}
& m_{r}{ }^{\prime}(t)=E\left[(t-X)^{r} / X<t\right]=\frac{1}{F(t)} \int_{0}^{t}(t-x)^{r} f(x ; \lambda, \sigma, \theta) d x \\
& =\frac{1}{F(t)}\left[\frac{1}{\lambda \sigma^{\theta}} \int_{0}^{t^{\theta}}\left(t-u^{1 / \theta}\right)^{r} e^{-u / \lambda \sigma^{\theta}} d u\right]
\end{aligned}
$$

Therefore:

$$
\begin{gathered}
m_{r}^{\prime}(t)=\frac{1}{1-e^{-\frac{1}{\lambda}\left(\frac{t}{\sigma}\right)^{\theta}} \sum_{j=0}^{r}(-1)^{j}\left(\begin{array}{l}
r \\
j
\end{array}\right) t^{r-j}} \\
\sigma^{j} \lambda^{j / \theta}\left[\Gamma\left(\frac{j}{\theta}+1, \lambda\right)-\Gamma\left(\frac{j}{\theta}+1, \frac{\lambda t^{\theta}}{\sigma^{\theta}}\right)\right]
\end{gathered}
$$

\section{L-Moments}

Suppose $X_{k: n}$ be the $k^{\text {th }}$ smallest moment in a sample of size $\mathrm{n}$, then the L-moments of $\mathrm{X}$ are defined by:

$$
l_{r}=\frac{1}{r} \sum_{k=0}^{r-1}(-1)^{k}\left(\begin{array}{c}
r-1 \\
k
\end{array}\right) E\left[X_{r-k: r}\right], \quad r=1,2, \ldots
$$

For OELLD with parameters $\lambda, \theta$ and $\sigma$, we have:

$$
\begin{aligned}
& E\left[X_{j: r}\right]=\frac{r !}{(j-1) !(r-j) !} \int_{0}^{\infty} x[F(x)]^{j-1}[1-F(x)]^{r-j} f(x) d x \\
& \Rightarrow E\left[X_{j: r}\right]=\frac{r !}{(j-1) !(r-j) !} \frac{\theta}{\lambda \sigma^{\theta}} \\
& \int_{0}^{\infty} x^{\theta}\left[1-e^{-\frac{1}{\lambda}\left(\frac{x}{\sigma}\right)^{\theta}}\right]^{j-1}\left[e^{-\frac{1}{\lambda}\left(\frac{x}{\sigma}\right)^{\theta}}\right]^{r-j} e^{-\frac{1}{\lambda}\left(\frac{x}{\sigma}\right)^{\theta}} d x
\end{aligned}
$$

Therefore, the first four L - Moments OELLD are:

$$
\begin{aligned}
& l_{1}=E\left[X_{1: 1}\right]=\sigma \lambda^{1 / \theta} \Gamma\left(\frac{1}{\theta}+1, \lambda\right) ; l_{2}=\frac{1}{2} E\left[X_{2: 2}-X_{1: 2}\right] \\
& =\sigma \lambda^{1 / \theta}\left[\Gamma\left(\frac{1}{\theta}+1, \lambda\right)-\frac{1}{2^{1 / \theta}} \Gamma\left(\frac{1}{\theta}+1,2 \lambda\right)\right] \\
& l_{3}=\frac{1}{3} E\left[X_{3: 3}-2 X_{2: 3}+X_{1: 3}\right] \\
& =\sigma \lambda^{1 / \theta}\left[\Gamma\left(\frac{1}{\theta}+1, \lambda\right)-\frac{3}{2^{1 / \theta}} \Gamma\left(\frac{1}{\theta}+1,2 \lambda\right)+\frac{2}{3^{1 / \theta}} \Gamma\left(\frac{1}{\theta}+1,3 \lambda\right)\right] \\
& l_{4}=\frac{1}{4} E\left[X_{4: 4}-3 X_{3: 4}+3 X_{2: 4}-X_{1: 4}\right] \\
& =\sigma \lambda^{1 / \theta}\left[\Gamma\left(\frac{1}{\theta}+1, \lambda\right)-\frac{6}{2^{1 / \theta}} \Gamma\left(\frac{1}{\theta}+1,2 \lambda\right)\right. \\
& {\left[+\frac{10}{3^{1 / \theta}} \Gamma\left(\frac{1}{\theta}+1,3 \lambda\right)-\frac{5}{4^{1 / \theta}} \Gamma\left(\frac{1}{\theta}+1,4 \lambda\right)\right]}
\end{aligned}
$$

\section{Quantile Function of OELLD}

The quantile function, say $Q(p)$, defined by $F(Q(p))$ $=p$ is the root of the equation:

$$
1-e^{-\frac{\lambda}{\sigma^{\theta}} Q(p)^{\theta}}=p \Rightarrow Q(p)=\sigma[-\ln (1-p) \lambda]^{1 / \theta}
$$

Thus, the $100_{\mathrm{qth}}$ percentiles are $t_{q}=\sigma[-\lambda \ln (1-q)]^{1 / \theta}$ and 50th percentiles is the median.

\section{Bonferroni Curve, Lorenz Curve and Gini's Index: $100_{\text {qth }}$}

The Bonferroni and Lorenz curves are respectively defined by:

$$
\begin{aligned}
B(p) & =\frac{1}{p \mu} \int_{0}^{q} x f(x ; \lambda, \sigma, \theta) d x \text { and } \\
\mathrm{L}(p) & =\frac{1}{\mu} \int_{0}^{q} x f(x ; \lambda, \sigma, \theta) d x
\end{aligned}
$$

or equivalently:

$$
B(p)=\frac{1}{p \mu} \int_{0}^{p} F^{-1}(x) d x \text { and } \mathrm{L}(p)=\frac{1}{\mu} \int_{0}^{p} F^{-1}(x) d x
$$

where, $\mu=E(x)$ and $q=F^{-1}(p)$.

The Bonferroni and Gini indices are:

$B=1-\int_{0}^{1} \mathrm{~B}(\mathrm{p}) d p$ and $G=1-2 \int_{0}^{1} \mathrm{~L}(\mathrm{p}) d p$

By using quantile function, we calculate the equations given in (13) as:

$$
\begin{aligned}
& \int_{0}^{p} F^{-1}(x) d x=-\sigma \int_{0}^{p}[\ln (1-x) \lambda] d x \\
& =\sigma e^{\lambda} \lambda^{1 / \theta}\left[\gamma\left(\frac{1}{\theta}+1\right)-\gamma\left(\frac{1}{\theta}+1, \ln (1-p)\right)\right]
\end{aligned}
$$

Then:

$$
B(p)=\frac{\sigma e^{\lambda} \lambda^{1 / \theta}}{p \mu}\left[\gamma\left(\frac{1}{\theta}+1\right)-\gamma\left(\frac{1}{\theta}+1, \ln (1-p)\right)\right]
$$

and:

$$
\mathrm{L}(p)=\frac{\sigma e^{\lambda} \lambda^{1 / \theta}}{\mu}\left[\gamma\left(\frac{1}{\theta}+1\right)-\gamma\left(\frac{1}{\theta}+1, \ln (1-p)\right)\right]
$$

Integrating Equation 15 and 16 with respect to $p$, we get the Bonferroni and Gini indices as: 


$$
B=1-\frac{\sigma e^{\lambda} \lambda^{1 / \theta}}{p \mu}\left[\gamma\left(\frac{1}{\theta}+1\right)-\int_{0}^{1} \gamma\left(\frac{1}{\theta}+1, \ln (1-p)\right) d p\right]
$$

and:

$$
G=\frac{2 \sigma e^{\lambda} \lambda^{1 / \theta}}{\mu}\left[\gamma\left(\frac{1}{\theta}+1\right)-\int_{0}^{1} \gamma\left(\frac{1}{\theta}+1, \ln (1-p)\right) d p\right]
$$

\section{Order Statistics}

Suppose $X_{1}, X_{2}, \ldots X_{n}$ is a random sample from the p.d.f of OELLD. Let $X_{(1)}, X_{(2)}, \ldots X_{(n)}$ be the corresponding order statistics. The probability density function and the cumulative distribution function of the $k^{\text {th }}$ order statistics, say $Y=X_{(k)}$ are:

$$
\begin{aligned}
& f_{Y}(y)=\frac{n !}{(k-1) !(n-k) !} F^{k-1}(y)[1-F(y)]^{n-k} f(y) \\
& =\frac{n !}{(k-1) !(n-k) ! \lambda \sigma^{\theta}} y^{\theta-1}\left[1-e^{-\frac{1}{\lambda}\left(\frac{y}{\sigma}\right)^{\theta}}\right]^{k-1}\left[e^{-\frac{1}{\lambda}\left(\frac{y}{\sigma}\right)^{\theta}}\right]^{n-k+1}
\end{aligned}
$$

and:

$$
\begin{aligned}
& F_{Y}(y)=\sum_{j=k}^{n}\left(\begin{array}{l}
n \\
j
\end{array}\right) F^{j}(y)[1-F(y)]^{n-j} \\
& =\sum_{j=k}^{n}\left(\begin{array}{l}
n \\
j
\end{array}\right)\left[1-e^{-\frac{1}{\lambda}\left(\frac{y}{\sigma}\right)^{\theta}}\right]^{j}\left[e^{-\frac{1}{\lambda}\left(\frac{y}{\sigma}\right)^{\theta}}\right]^{n-j}
\end{aligned}
$$

\section{Reliability and Related Properties}

The reliability function and hazard rate function of OELLD are:

$$
R(x)=e^{-\frac{1}{\lambda}\left(\frac{x}{\sigma}\right)^{\theta}} \text { and } h(x)=\frac{\theta}{\lambda \sigma^{\theta}} x^{\theta-1}
$$

Now:

$$
\begin{aligned}
& \text { Now, } f_{e . l l}(x ; \lambda, \sigma, \theta)=\frac{\theta}{\lambda \sigma^{\theta}} x^{\theta-1} e^{-\frac{1}{\lambda}\left(\frac{x}{\sigma}\right)^{\theta}} \Rightarrow \ln f(x) \\
& =-\ln \lambda+\ln \theta-\theta \ln \sigma+(\theta-1) \ln x-\frac{1}{\lambda}\left(\frac{x}{\sigma}\right)^{\theta} \\
& \frac{d \ln f(x)}{d x}=\frac{\theta-1}{x}-\frac{1}{\lambda \sigma^{\theta}} \theta x^{\theta-1} \text { and } \frac{d^{2} \ln f(x)}{d^{2} x} \\
& =-(\theta-1)\left[\frac{1}{x^{2}}+\frac{1}{\lambda \sigma^{\theta}} \theta x^{\theta-2}\right]
\end{aligned}
$$

The distribution is log-concave for $\lambda>0, \theta>1, \sigma>0$ and $x>0, \frac{d^{2} \ln f(x)}{d^{2} x}<0$. Therefore, the distribution satisfies the properties of Increasing Failure Rate (IFR) and Decreasing Mean Residual Life (DMRL).

Mean residual life (MRL) is:

$$
\begin{aligned}
& e_{x}(t)=\frac{1}{e^{\frac{-1}{\lambda}\left(\frac{t}{\sigma}\right)^{\theta}}} \sum_{j=0}^{1}(-1)^{j}\left(\begin{array}{l}
1 \\
j
\end{array}\right) t^{1-j} \sigma^{j} \lambda^{\frac{j}{\theta}} \Gamma\left(\frac{j}{\theta}+1, \frac{\lambda t^{\theta}}{\sigma^{\theta}}\right) \\
& =\frac{1}{e^{\frac{-1}{\lambda}\left(\frac{t}{\sigma}\right)^{\theta}}}\left[t \Gamma\left(1, \frac{\lambda t^{\theta}}{\sigma^{\theta}}\right)-\frac{\sigma}{\lambda^{\frac{1}{\theta}}} \Gamma\left(\frac{1}{\theta}+1, \frac{\lambda t^{\theta}}{\sigma^{\theta}}\right)\right]
\end{aligned}
$$

Reversed hazard rate:

$m(x)=\frac{f(x)}{F(x)}=\frac{\frac{\theta}{\lambda \sigma^{\theta}} x^{\theta-1} e^{\left.-\frac{1}{\lambda} \frac{x}{\sigma}\right)^{\theta}}}{1-e^{-\frac{1}{\lambda}\left(\frac{x}{\sigma}\right)^{\theta}}}$

Mean Reversed Residual Life (MRRL) function or Expected Inactivity Time (EIT) is defined as:

$$
\begin{aligned}
& \overline{\mathrm{e}}_{\mathrm{x}}(\mathrm{t})=\mathrm{E}((\mathrm{t}-\mathrm{x}) / \mathrm{x}<\mathrm{t})=\frac{1}{1-\mathrm{e}^{-\frac{1}{\lambda}\left(\frac{\mathrm{t}}{\sigma}\right)^{\theta}} \sum_{\mathrm{j}=0}^{1}(-1)^{\mathrm{j}}\left(\begin{array}{l}
1 \\
\mathrm{j}
\end{array}\right) \mathrm{t}^{1-\mathrm{j}}} \\
& \sigma^{\mathrm{j}} \lambda^{\frac{\mathrm{j}}{\theta}}\left[\Gamma\left(\frac{\mathrm{j}}{\theta}+1, \lambda\right)-\Gamma\left(\frac{\mathrm{j}}{\theta}+1, \frac{\lambda \mathrm{t}^{\theta}}{\sigma^{\theta}}\right)\right] \\
& =\frac{1}{1-\mathrm{e}^{-\frac{1}{\lambda}\left(\frac{\mathrm{t}}{\sigma}\right)^{\theta}}}\left[\mathrm{t}\left\{\Gamma(1, \lambda)-\Gamma\left(1, \frac{\lambda \mathrm{t}^{\theta}}{\sigma^{\theta}}\right)\right\}\right. \\
& \left.-\frac{\sigma}{\lambda^{\frac{1}{\theta}}}\left\{\Gamma\left(\frac{1}{\theta}+1, \lambda\right)-\Gamma\left(\frac{1}{\theta}+1, \frac{\lambda \mathrm{t}^{\theta}}{\sigma^{\theta}}\right)\right\}\right]
\end{aligned}
$$

\section{Estimation of the Parameters}

Using the method of ML method of Estimation (MLE), we estimate the parameters of the OELLD. The likelihood function is:

$$
\begin{aligned}
& L(x ; \lambda, \theta, \sigma)=\prod_{i=1}^{n} f\left(x_{i} ; \lambda, \theta, \sigma\right) \\
& =\prod_{i=1}^{n} \frac{\theta}{\lambda \sigma^{\theta}} x_{i}^{\theta-1} e^{-\frac{1}{\lambda}\left(\frac{x_{i}}{\sigma}\right)^{\theta}} \\
& \Rightarrow \ln L=-n \ln \lambda+n \ln \theta-\theta n \ln \sigma \\
& +(\theta-1) \sum_{i=1}^{n} \ln x_{i}-\frac{1}{\lambda \sigma^{\theta}} \sum_{i=1}^{n} x_{i}^{\theta}
\end{aligned}
$$

The ML equations of $\lambda, \sigma$ and $\theta$ are:

$$
\begin{aligned}
& \frac{\partial \ln L}{\partial \lambda}=\frac{-n}{\lambda}+\frac{1}{\lambda^{2} \sigma^{\theta}} \sum_{i=1}^{n} x_{i}^{\theta}=0 ; \frac{\partial \ln L}{\partial \sigma}=\frac{-\theta n}{\sigma}+\frac{\theta}{\lambda \sigma^{\theta+1}} \sum_{i=1}^{n} x_{i}^{\theta}=0 \\
& \text { and } \frac{\partial \ln L}{\partial \theta}=\frac{n}{\theta}-n \ln \sigma+\sum_{i=1}^{n} \ln x_{i}-\frac{1}{\lambda} \sum_{i=1}^{n}\left(\frac{x_{i}}{\sigma}\right)^{\theta} \ln \left(\frac{x_{i}}{\sigma}\right)=0
\end{aligned}
$$



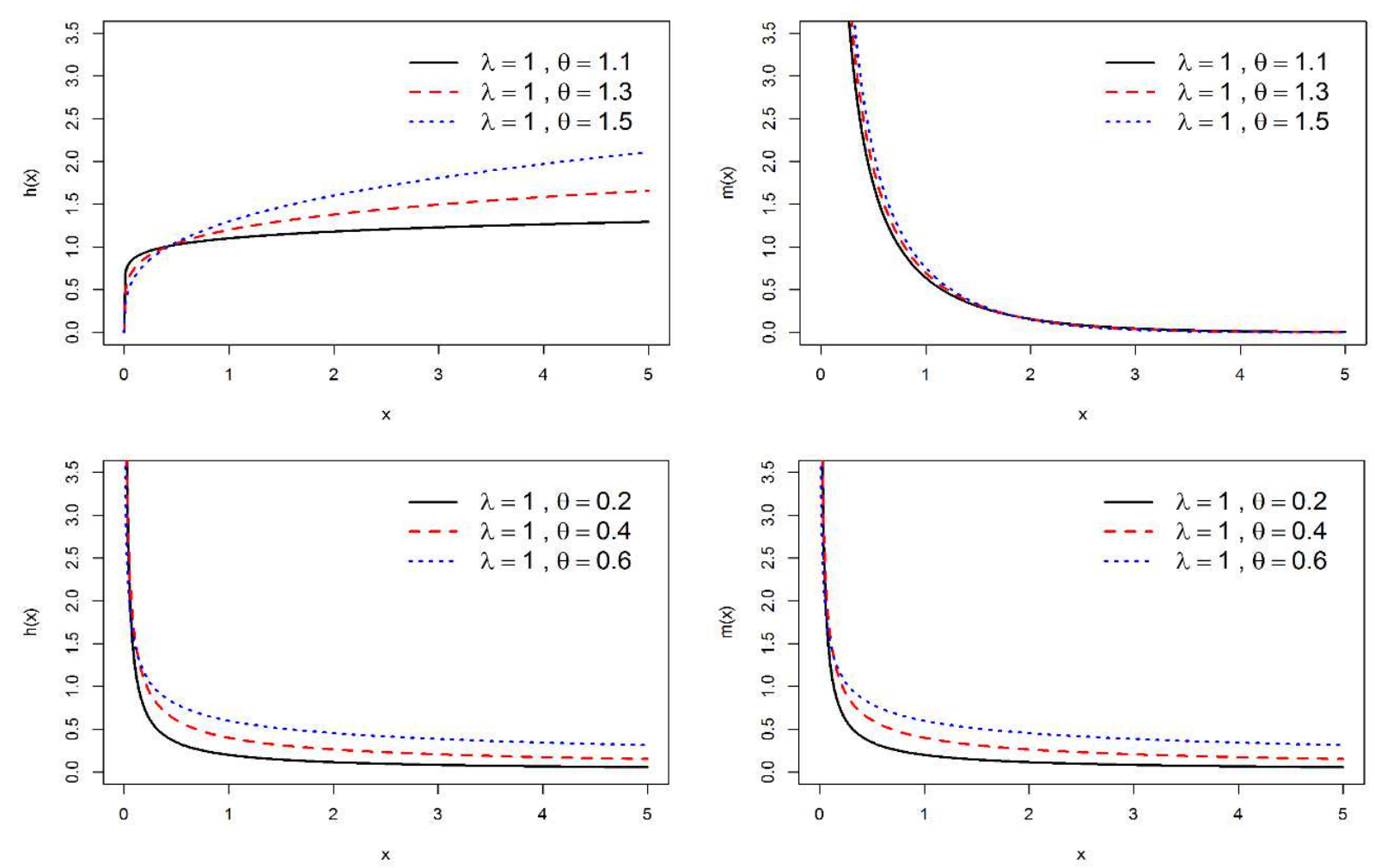

Fig. 3. Hazard rate and reversed hazard rate of the OELLD for different $\lambda$ and $\theta$ at $\sigma=1$

ML estimates of the parameters $\lambda, \sigma$ and $\theta$ can be obtained by simultaneously solving the above three equations using some numerical iterative techniques.

Second derivatives of MLEs are:

$$
\begin{aligned}
& \frac{\partial^{2} \ln L}{\partial \lambda^{2}}=\frac{n}{\lambda^{2}}-\frac{2}{\lambda^{3}} \sum_{i=1}^{n}\left(\frac{x_{i}}{\sigma}\right)^{\theta} ; \frac{\partial^{2} \ln L}{\partial \sigma^{2}}=\frac{n \theta}{\sigma^{2}}-\frac{\theta(\theta+1)}{\lambda \sigma^{\theta+2}} \sum_{i=1}^{n} x_{i}^{\theta} \\
& \text { and } \frac{\partial^{2} \ln L}{\partial \theta^{2}}=-\frac{n}{\theta^{2}}-\frac{1}{\lambda} \sum_{i=1}^{n}\left(\frac{x_{i}}{\sigma}\right)^{\theta}\left[\ln \left(\frac{x_{i}}{\sigma}\right)\right]^{2} \\
& \frac{\partial^{2} \ln L}{\partial \lambda \partial \theta}=\frac{1}{\lambda^{2}} \sum_{i=1}^{n}\left(\frac{x_{i}}{\sigma}\right)^{\theta} \ln \left(\frac{x_{i}}{\sigma}\right) ; \frac{\partial^{2} \ln L}{\partial \lambda \partial \sigma}=-\frac{\theta}{\lambda^{2} \sigma} \sum_{i=1}^{n}\left(\frac{x_{i}}{\sigma}\right)^{\theta} \\
& \text { and } \frac{\partial^{2} \ln L}{\partial \sigma \partial \theta}=-\frac{n}{\sigma}-\frac{1}{\lambda \sigma} \sum_{i=1}^{n}\left(\frac{x_{i}}{\sigma}\right)^{\theta}\left[\theta \ln \left(\frac{x_{i}}{\sigma}\right)+1\right]
\end{aligned}
$$

\section{Data Analysis}

In this section, we study the application of the OELLD (and their sub-models: ELLog, LeLLog and LLog distributions considered by Lemonte (2014)) for a real lifetime data set to illustrate its potentiality. The following real lifetime data set corresponds to an uncensored data set from Nichols and Padgett (2006) on breaking stress of carbon fibres (in Gba):

$3.70,2.74,2.73,2.50,3.60,3.11,3.27$, $2.87,1.47,3.11,4.42,2.41,3.19,3.22,1.69$,
$3.28,3.09,1.87,3.15,4.90,3.75,2.43$, $2.95,2.97,3.39,2.96,2.53,2.67,2.93,3.22$, $3.39,2.81,4.20,3.33,2.55,3.31,3.31$, $2.85,2.56,3.56,3.15,2.35,2.55,2.59,2.38$, $2.81,2.77,2.17,2.83,1.92,1.41,3.68$, $2.97,1.36,0.98,2.76,4.91,3.68,1.84,1.59$, $3.19,1.57,0.81,5.56,1.73,1.59,2.00$, $1.22,1.12,1.71,2.17,1.17,5.08,2.48,1.18$, $3.51,2.17,1.69,1.25,4.38,1.84,0.39$, $3.68,2.48,0.85,1.61,2.79,4.70,2.03,1.80$, $1.57,1.08,2.03,1.61,2.12,1.89,2.88$, $2.82,2.05,3.65$.

We fitted the proposed OELLD curve for the above data, which is shown in the following graphs:

The unknown parameters of OELLD are estimated by the ML method. In order to compare the models considered by Lemonte (2014) with the proposed OELLD model, the Cramér-von Mises $\left(\mathrm{W}^{*}\right)$ and Anderson-Darling $\left(\mathrm{A}^{*}\right)$ statistics are used. The details of the statistics $\mathrm{W}^{*}$ and $\mathrm{A}^{*}$ are described in Chen and Balakrishnan (1995). In general, the smaller the values of these statistics, the better the fit to the data. Let $\mathrm{H}(\mathrm{x} ; \theta)$ be the c.d.f., where the form of $\mathrm{H}$ is known but $\theta$ (a k-dimensional parameter vector) is unknown. The statistics $\mathrm{W}^{*}$ and $\mathrm{A}^{*}$ can be obtained as follows: 
- Compute $v_{i}=H\left(x_{i} ; \hat{\theta}\right)$, where the $\mathrm{x}_{\mathrm{i}}$ 's are in ascending order and then $y_{i}=\phi^{-1}\left(v_{i}\right)$, where $\phi(\cdot)$ is the standard normal c.d.f. and $\phi^{-1}(\cdot)$ its inverse

- Compute $u_{i}=\phi\left\{\left(y_{i}-\bar{y}\right) / s_{y}\right\}$, where $\bar{y}=(1 / n) \sum_{i=1}^{n} y_{i}$ and $\mathrm{s}_{\mathrm{y}}^{2}=(n-1)^{-1} \sum_{i=1}^{n}\left(y_{i}-\bar{y}\right)^{2}$

- Calculate $W^{2}=\sum_{i=1}^{n}\left\{u_{i}-(2 i-1) /(2 n)\right\}^{2}+1 /(12 n)$ and $\mathrm{A}^{2}=-n-(1 / n) \sum_{i=1}^{n}\left\{(2 i-1) \ln \left(u_{i}\right)\right.$

$\left.+(2 n+1-2 i) \ln \left(1-u_{i}\right)\right\}$ and then $W^{*}=W^{2}(1+0.5 / n)$ and $\mathrm{A}^{*}=A^{2}\left(1+0.75 / n+2.25 / n^{2}\right)$

The Table 1 lists the MLEs (and the corresponding standard errors in parentheses) of the parameters of all the models for the data set (breaking stress of carbon fibres). The statistics $\mathrm{W}^{*}$ and $\mathrm{A}^{*}$ are also listed in this Table 1 for the models. It can be seen from the table given below, the proposed OELLD has the smallest values for the statistics $\mathrm{W}^{*}$ and $\mathrm{A}^{*}$ than most of the other models, that is, the proposed model fits the breaking stress of carbon fibres data better than most of the other models considered. More information is provided by a visual comparison in Fig. 4 of the histogram of the data with the fitted OELLD density function. Clearly, the OELLD distribution provides a closer fit to the histogram. The Kaplan-Meier (K-M) estimate and the estimated survival function of the fitted OELLD distribution is shown in Fig. 4. OELLD has three parameters only. From this plot, note that the OELLD model fits the data adequately and hence can be adequate for this data.
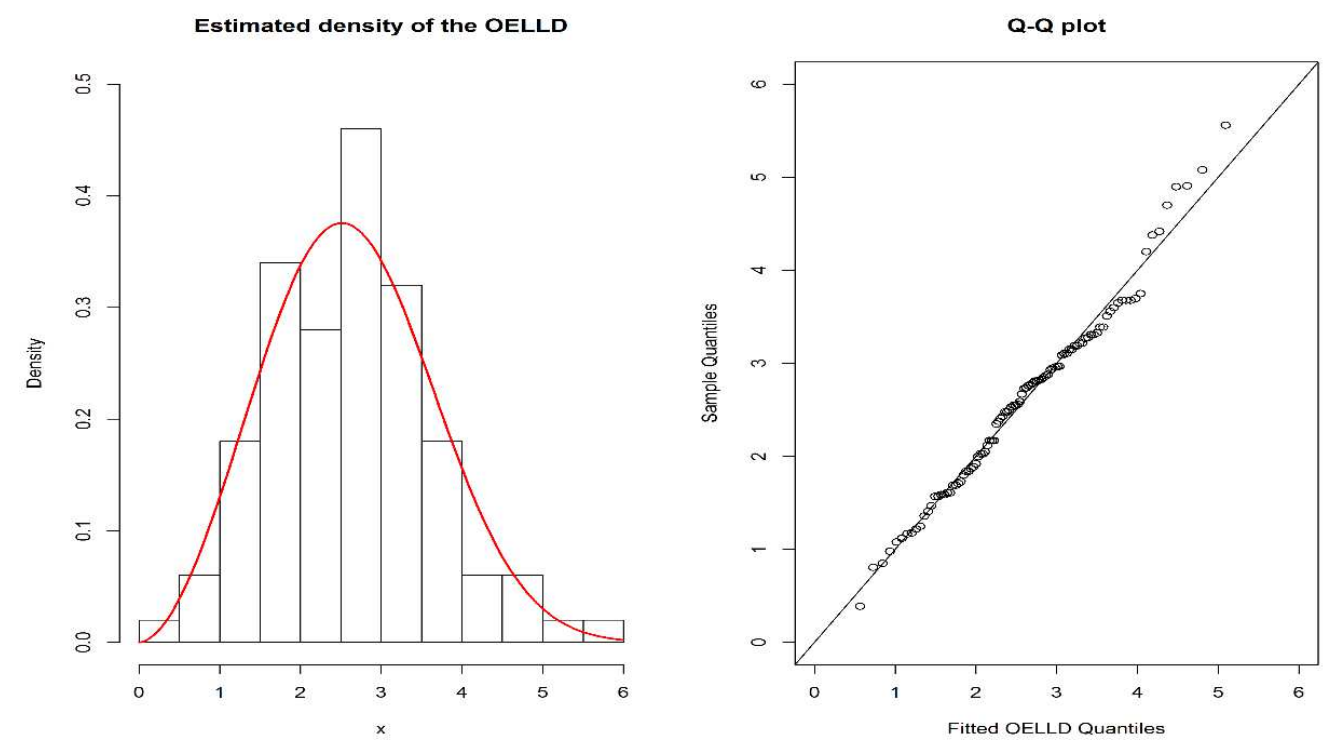

Fig. 4. Estimated density and Q-Q plot for OELLD

Table 1. MLEs (standard errors in parentheses) and the statistics $W^{*}$ and $A^{*}$ for the above data

\begin{tabular}{|c|c|c|c|c|c|c|}
\hline Distribution & Estimates & $W^{*}$ & $A^{*}$ & & & \\
\hline Beta log logistic $(a, b, \alpha, \beta)$ & $0.09(0.1700)$ & $0.2254(0.4452)$ & $3.1486(0.1851)$ & $25.417(46.670)$ & 0.03867 & 0.27763 \\
\hline $\operatorname{OELLD}(\sigma, \lambda, \theta)$ & $2.3743(6.3663)$ & $1.8227(13.6484)$ & $2.7931(0.2145)$ & & 0.0622 & 0.4158 \\
\hline Exponentiated log logistic $(a, \alpha, \beta)$ & $0.3339(0.0998)$ & $3.3815(0.2270)$ & $7.4714(1.4975)$ & & 0.04627 & 0.3019 \\
\hline $\operatorname{LeLLog}(b, \alpha, \beta)$ & $7.8795(11.370)$ & $5.6426(3.3334)$ & $3.0234(0.3873)$ & & 0.06717 & 0.38989 \\
\hline Exponentiated Weibull $(\alpha, \beta, a)$ & $0.0928(0.0904)$ & $2.4091(0.5930)$ & $1.3168(0.5969)$ & & 0.07036 & 0.41313 \\
\hline Marshall-Olkin Weibull $(\alpha, \gamma, \lambda)$ & $0.6926(0.8310)$ & $3.0094(0.7181)$ & $0.0309(0.0472)$ & & 0.07052 & 0.43016 \\
\hline \multicolumn{7}{|l|}{ Beta half-Cauchy } \\
\hline$(\varphi, a, b)$ & $15.194(20.687)$ & $5.5944(0.8087)$ & $46.116(70.775)$ & & 0.1386 & 0.70838 \\
\hline $\log \operatorname{Logistic}(\alpha, \beta)$ & $2.4984(0.1051)$ & $4.1179(0.3444)$ & & & 0.23903 & 1.2409 \\
\hline Weibull $(\alpha, \beta)$ & $0.049(0.0138)$ & $2.7929(0.2131)$ & & & 0.06227 & 0.41581 \\
\hline $\operatorname{Gamma}(\lambda, \eta)$ & $5.9526(0.8193)$ & $2.2708(0.3261)$ & & & 0.14802 & 0.75721 \\
\hline $\log -\operatorname{normal}(\mu, \sigma)$ & $0.8774(0.0444)$ & $0.4439(0.0314)$ & & & 0.27734 & 1.48332 \\
\hline $\operatorname{Birnbaum-Saunders}(\alpha, \beta)$ & $0.4622(0.0327)$ & $2.366(0.1064)$ & & & 0.29785 & 1.61816 \\
\hline
\end{tabular}


The estimated variance-covariance matrix is:

$$
\begin{aligned}
& I^{-1}(\theta)=\left[\begin{array}{lll}
\frac{\partial^{2} \ln L}{\partial \sigma^{2}} & \frac{\partial^{2} \ln l}{\partial \sigma \partial \lambda} & \frac{\partial^{2} \ln l}{\partial \sigma \partial \theta} \\
\frac{\partial^{2} \ln L}{\partial \lambda \partial \sigma} & \frac{\partial^{2} \ln l}{\partial \lambda^{2}} & \frac{\partial^{2} \ln l}{\partial \lambda \partial \theta} \\
\frac{\partial^{2} \ln L}{\partial \theta \partial \sigma} & \frac{\partial^{2} \ln l}{\partial \theta \partial \lambda} & \frac{\partial^{2} \ln l}{\partial \theta^{2}}
\end{array}\right]^{-1} \\
& =\left[\begin{array}{ccc}
138.4137 & 64.5495 & -43.6248 \\
64.5494 & -30.1081 & 20.3428 \\
-43.6248 & 20.3428 & -35.5612
\end{array}\right]^{-1} \\
& =\left[\begin{array}{ccc}
40.530 & 86.878 & 0.021 \\
-86.876 & 186.278 & -0.015 \\
0.026 & -0.024 & 0.046
\end{array}\right]
\end{aligned}
$$

\section{Concluding Remarks}

In this study, we proposed a new probability distribution and named it as Odds Exponential Log Logistic Distribution (OELLD). This distribution is a particular case of T-X family of distributions proposed by Alzaatreh et al. (2013). The structural properties of this distribution have been studied and inferences on parameters have also been mentioned. The advantage of this proposed distribution is, it has only three parameters that are to be estimated. The appropriateness of fitting the odds exponential log logistic distribution has been established by analyzing a real lifetime data set.

\section{Acknowledgement}

The authors are very much thankful to the reviewers and the editor for their valuable suggestions to improve the quality of this manuscript.

The authors K. Kalyani and D.C.U. Sivakumar are indebted to UGC under BSR scheme for providing research grant.

\section{Author's Contributions}

Rosaiah Kanaparthi: Wrote section 1.

Gadde Srinivasa Rao: Wrote section 4.

Kruthiventi Kalyani: Wrote section 3.

Devireddy Charana Udaya Sivakumar: Wrote section 2.

\section{Ethics}

This article is original and contains unpublished material. The corresponding author confirms that all of the other authors have read and approved the manuscript and no ethical issues involved.

\section{References}

Akinsete, A., F. Famoye and C. Lee, 2008. The betaPareto distribution. J. Theor. Applied Stat., 42: 547-563. DOI: 10.1080/02331880801983876

Akinsete, A.A. and C. Lowe, 2009. The beta-Rayleigh distribution in reliability measure. Statistics, 43: 3103-3107.

Aljarrah, M.A., C. Lee and F. Famoye, 2014. On generating $\mathrm{T}-\mathrm{X}$ family of distributions using quantile functions. J. Stat. Distribut. Applic., 1: 1-17. DOI: $10.1186 / 2195-5832-1-2$

Alzaatreh, A., C. Lee and F.A. Famoye, 2013. A new method for generating families of continuous distributions. Metron, 71: 63-79. DOI: $10.1007 / \mathrm{s} 40300-013-0007-y$

Ashkar, F. and S. Mahdi, 2006. Fitting the log-logistic distribution by generalized moments. J. Hydrol., 328: 694-703. DOI: 10.1016/j.jhydrol.2006.01.014

Box, G. and D. Cox, 1964. An analysis of transformations. J. Royal Stat. Society, 26; 211-246.

Chen, G. and N. Balakrishnan, 1995. A general purpose approximate goodness-of-fit test. J. Quality Technol., 27: 154-161.

Cox, D., 1972. Regression models and life-tables. J. Royal Stat. Society, 34: 187-200.

El-Damcese, M.A., A. Mustafa, B.S. El-Desouky and M.E. Mustafa, 2015. Odd generalized exponential gompertz. Statistics Theory.

Eugene, N., C. Lee and F. Famoye, 2002. Beta-normal distribution and its applications. Commun. Stat. Theory Meth., 31: 497-512. DOI: 10.1081/STA-120003130

Famoye, F., C. Lee and O. Olumolade, 2005. The betaWeibull distribution. J. Stat. Theory Applied, 4: 121-136.

Gupta, R.C. and R.D. Gupta, 2007. Proportional reversed hazard rate model and its applications. J. Stat. Plann. Inference, 137: 3525-3536. DOI: $10.1016 /$ j.jspi.2007.03.029

Gupta, R.D. and D. Kundu, 1999. Theory and methods: Generalized exponential distribution. Austral. New Zealand J. Stat., 41: 173-188. DOI: $10.1111 / 1467-842 X .00072$

Kantam, R.R.L. and G.S. Rao, 2002. Log-logistic distribution: Modified maximum likelihood estimation. Gujarat Stat. Rev., 29: 25-36.

Kozubowski, T.J. and S. Nadarajah, 2008. The beta Laplace distribution. J. Comput. Anal. Applic., 10: 305-318.

Lemonte, A.J., 2014. The beta log-logistic distribution. Brazil. J. Probability Stat., 28: 313-332. DOI: $10.1214 / 12-B J P S 209$

Marshall, A.W. and I. Olkin, 1997. A new method for adding a parameter to a family of distributions with application to the exponential and Weibull families. Biometrika, 84: 641-652.

DOI: $10.1093 /$ biomet $/ 84.3 .641$ 
Nadarajah, S. and S. Kotz, 2004. The beta Gumbel distribution. Math. Prob. Eng., 4: 323-332. DOI: $10.1155 / \mathrm{S} 1024123 \mathrm{X} 04403068$

Nadarajah, S. and S. Kotz, 2006. The beta exponential distribution. Stat. Reliability Eng. Syst. Safety, 91: 689-697. DOI: 10.1016/j.ress.2005.05.008

Nichols, M.D. and W.J. Padgett, 2006. A Bootstrap control chart for Weibull percentiles. Quality Reliability Eng. Int., 22: 141-151.

DOI: 10.1002 /qre.691
Rosaiah, K., R.R.L. Kantam and C.S. Kumar, 2006. Reliability test plans for exponentiated log-logistic distribution. Economic Quality Control, 21: 279-28. DOI: $10.1515 /$ EQC.2006.279

Tahir, M.H., G.M. Cordeiro, M. Alizadeh, M. Mansoor and M. Zubair et al., 2015. The odd generalized exponential family of distributions with applications. J. Stat. Distribut. Applic., 2: 1-28. DOI: 10.1186/s40488-014-0024-2 